\title{
Die Benzoylverbindungen der bei der Spaltung der Eiweisskörper entstehenden Amidosäuren.
}

\author{
Von
}

Dr. Albert Schultze.

(Aus der chemischen Abtheilung des physiologischen Instituts zu Berlin.

(Der Redaction zugegangen am 15. Mai 1900.)

Bei der hydrolytischen Spaltung der Eiweisskörper, wie sie durch Einwirkung von Mineralsäuren und Alkalien in der Hitze, einigen Enzymen, speziell durch das Trypsin des Pankreas sowie durch Mikroorganismen bewirkt wird, entsteht regelmässig eine Reihe von Amidosäuren. Die Methoden; welche zum Isoliren dieser Säuren und zu ihrer Reindarstellung zur Verfügung stehen, sind ausserordentlich umständlich und zeitraubend und weit davon entfernt, quantitativ zu sein. Gelegentlich einer Untersuchung über die Trypsinverdauung des Leims, welche ich vor einigen Semestern ausführte, hatte ich selbst Gelegenheit, diesen Mangel zu empfinden. Ich folgte daher gern der Aufforderung des Herrn Professor Dr. Thierfelder, nach Verbindungen der Amidosäuren $\mathrm{zu}$ suchen, welche charakteristisch und besser als die bisher bekannten für die Isolirung und Trennung sich erwiesen. Es lag nahe, an die Benzoylverbindungen $\mathrm{zu}$ denken, umsomehr, als die Ueberführung des Glycocolls in die schwerlösliche Hippursäure bereits mit Erfolg zur Abscheidung dieser leichtlöslichen Amidosäure, welche bei der Spaltung des Leims und anderer Albuminoide in reichlicher Menge auftritt, ${ }^{1}$ ) benutzt worden ist. ${ }^{2}$ )

1) Nach den Untersuchungen von K. Spiro (Zeitschr. f. phys. Chem., Bd. XXVIII, S. 174) entsteht Glykokoll in geringer Menge auch bei der Zersetzung mancher Eiweissstoffe.

2) Charles S. Fischer, Zeitschr. f. phys. Chem., Bd. XIX., S. 175. Max Gonnermann, Arch. f. d. ges. Physiol., Bd. 59, S. 43. S. 309 .

Edwin S. Faust, Arch. f. exper. Pathol. u. Pharmak., Bd. 41, 
Als Benzoylirungsmittel benutzt man jetzt vielfach das Benzoylchlorid, welches unter gewissen Bedingungen mit Alkoholen, Phenolen, primären und secundären Aminen, Diaminen in der Weise reagirt, dass Benzoesäureester, resp. substituirte Benzamide entstehen. In sehr bequemer Weise lässt sich die Benzoylirung in vielen Fällen ausführen, wenn man die betreffende Substanz in wässeriger Lösung mit Natronlauge und Benzoylchlorid schüttelt (Schotten-Baumann'sche Reaction). Der Erste, welcher die Reaction auf Amidosäuren anwandte, war Baum. ${ }^{1}$ ) Er löste Glycocoll in wenig Wasser, fügte einige Tropfen Natronlauge hinzu, schüttelte mit Benzoylchlorid, welches allmählich im Ueberschuss zugesetzt wurde, und machte schliesslich mit Natronlauge alkalisch. Er fand, dass Glycocoll fast vollständig in Hippursäure übergeht. Um die Hippursäure zu isoliren, wurde die alkalische Flüssigkeit mit einer Mineralsäure angesäuert und dem abgeschiedenen Gemenge von Benzoesäure und Hippursäure mit Aether die Benzoesäure entzogen. In derselben Weise führte Baum die Benzoylirung des Alanins aus. Das durch Salzsäure abgeschiedene Benzoylalanin wurde zur Entfernung der Benzoesäure wiederholt mit Petroläther erhitzt, der Rückstand mit wenig Aether gewaschen und aus heissem. Aether umkrystallisirt. Es schieden sich weisse glänzende Blättchen aus. Ueber die anderen uns hier interessirenden Amidosäuren der aliphatischen Reihe macht er keine näheren Angaben. Er sagt nur, «andere Amidosäuren zeigen ein ähnliches Verhalten wie das Glycocoll». Dagegen hat Baum das Benzoylirungsverfahren auch auf das Tyrosin angewandt. Es zeigten sich hier andere Verhältnisse. Die Untersuchung führte zu keinem befriedigenden Resultat.

Später hat Charles S. Fischer ${ }^{2}$ ) versucht, mit Hülfe der Schotten-Baumann'schen Methode die Benzoylverbindungen des Leucins und der Glutaminsäure darzustellen. Er versetzte die in überschüssiger $10 \%$ iger Natronlauge gelöste Substanz mit Benzoylchlorid unter beständigem Umschütteln so

1) Diese Zeitschr., Bd. IX, S. 465 .

2) a. a. 0 . 
lange in kleinen Portionen, bis der Geruch des letzteren nicht mehr verschwand. Nach dem Ansäuern mit Salzsäure wurde mit Aether ausgeschüttelt, der Aether abdestillirt und der Aetherrückstand durch längeres Kochen mit Petroläther von der Benzoesäure befreit. Es hinterblieb eine syrupförmige Masse, welche in kaltem Wasser unlöslich, in heissem Wasser, Alkohol und Chloroform löslich war und nicht zur Krystallisation gebracht werden konnte. Von dem aus Glutaminsäure erhaltenen Körper gibt Charles S. Fischer an, dass er in viel heissem Wasser gelöst sich beim Erkalten als Oel wieder abscheide. Die Anwendung des Schotten-Baumann'schen Verfahrens auf die als Zersetzungsprodukte der Eiweisskörper bekannten Amidosäuren, Leucin, Asparaginsäure, Glutaminsäure, Tyrosin hat also bisher kein positives Resultat ergeben. Das Alanin kommt weniger in Betracht, da es bisher nur bei der Spaltung von Seide nachgewiesen worden ist.

Als meine eigenen Versuche, deren Resultat im Folgenden mitgetheilt werden soll, nahezu abgeschlossen waren, erschien eine Arbeit von E. Fischer ${ }^{2}$ ) "Ueber die Spaltung einiger racemischer Amidosäuren in die optisch-activen Componenten». Um die bei der hydrolytischen Spaltung der Eiweissstoffe auftretenden optisch-activen Amidosäuren synthetisch herzustellen, hat E. Fischer zunächst die künstlichen (racemischen) Amidosäuren in ihre Benzoylderivate verwandelt, diese mit Hülfe ihrer Alkaloid- (Brucin, Strychnin) Salze getrennt und aus den auf diese Weise isolirten optischen Antipoden durch Kochen mit Säure die optisch-activen Amidosäuren erhalten. Er nahm die Benzoylirung bei Gegenwart von Natriumbicarbonat vor und erzielte hiermit sehr gute Ausbeute, während ihm die Anwendung von Alkali nur ganz unbefriedigende Resultate ergab. Auf die Ergebnisse der Untersuchungen von E. Fischer werde ich bei der Beschreibung meiner Versuche eingehen. Sie ermöglichten mir eine Deutung eigenthümlicher Beob-

1) Th. Weyl, Berichte d. d. ch. Ges., Bd. XXI, S. 1529.

2) Berichte d. d. ch. Ges., Bd. XXXII, S. 2451. 
achtungen, die ich bei der Benzoylirung der Glutaminsäure machte.

\section{Experimenteller Theil.}

Meine Untersuchungen erstrecken sich auf Asparaginsäure, Leucin, Glutaminsäure und Tyrosin. Die letzten drei wurden von mir aus Casein gewonnen. Die Spaltung geschah nach der Vorschrift von R. Cohn ${ }^{1}$ ) durch fünfstündiges Kochen mit der dreifachen Menge rauchender Salzsäure am Rückflusskühler, die Isolirung der Amidosäuren im Wesentlichen nach den Angaben von Hlasiwetz und Habermann. ${ }^{2}$ ) Die Asparaginsäure wurde von Kahlbaum bezogen.

\section{Benzoylleucin.}

Die Methoden, welche Baum beim Glycocoll und Alanin anwandte, sowie diejenige, welche Charles S. Fischer beim Leucin und bei der Glutaminsäure versuchte, führten zu keinem Resultat. Es hinterblieben nach dem Auskochen der Benzoesäure nur ölige oder syrupöse Massen, die nicht zur Krystallisation gebracht werden konnten. Ein besseres Ergebniss wurde erzielt, als ich die Benzoylirung in schwach alkalischer Lösung vornahm. $1 \mathrm{~g}$ Leucin wurde in einer gut schliessenden Stöpselflasche in 12\% oiger Natronlauge gelöst und die Lösung unter beständigem Schütteln mit im Ganzen $5 \mathrm{ccm}$. Benzoylchlorid in einzelnen kleinen Portionen in der Weise versetzt, dass ein neuer Zusatz erst dann erfolgte, wenn der Benzoylchloridgeruch vollständig verschwunden war. Sobald die alkalische Reaction umschlug und sich krystallinische Ausscheidungen. zeigten, brachte ich diese durch einen geringen Ueberschuss von Natronlauge in Lösung. Anfänglich vollzieht sich die Reaction ohne Wärmeentwicklung, nach kurzer Zeit jedoch tritt geringe Erwärmung ein. Nach ungefähr 40 Minuten ist die obèn

1) Diese Zeitschr., Bd. XXII, S. 161.

2) Liebig's Annalen, Bd. 1.69, S. 150. 
angegebene Menge Benzoylchlorid verbraucht. Nach dem Ansäuern mit concentrirter Salzsäure wird mit einer grossen Menge Aether wiederholt ausgeschüttelt. Bei dem Verdunsten der vereinigten ätherischen Lösungen in einer geräumigen Schale hinterbleibt eine weisse krystallinische Masse, die beim Erwärmen auf dem Dampfbade nach kurzer Zeit den grösseren Theil der Benzoesäure in zarten Kryställchen heraustreten lässt. Diese Krystalle können bequem durch gelindes Blasen mit dem Munde entfernt werden. Von Zeit zu Zeit löst man den Rest wieder in Aether, verdunstet diesen abermals und schafft in derselben Weise die aufs Neue herausgetretenen Efflorescenzen der Benzoesäure fort. In dem Maasse wie letztere verschwindet, wird die Krystallmasse dünnflüssig, bis schliesslich in der Schale ein Oel zurückbleibt, das nur noch eine ganz unbedeutende Menge von Benzoesäure einschliesst. Jetzt wurde das Oel in viel Wasser gelöst und die Lösung etwas concentrirt. Beim Erkalten schied sich Benzoylleucin in Form prismatischer Nadeln oder in Blättchen ab. Ausbeute $70 \%$ der Theorie. Die Trennung von Benzoylleucin und Benzoesäure lässt sich auch in folgender Weise bewerkstelligen. Der durch Salzsäure erhaltene Niederschlag wird abgesaugt, mit Wasser gewaschen, im Trockenschrank getrocknet, fein zerrieben und zur Entfernung der Benzoesäure im Kolben wiederholt mit Petroläther ausgekocht. Der Rückstand, der bei dieser Behandlung keine ölige, sondern eine krystallinische Beschaffenheit zeigte, wurde in der Wärme mit Aether wiederholt geschüttelt. Beim Verdunsten verbleibt ein weisser krystallinischer Rückstand, der in heissem Wasser gelöst wieder in derben prismatischen Nadeln oder Blättchen krystallisirte. Ausbeute $78 \%$. Beim Behandeln der Krystalle mit einer zur Lösung unzureichenden Menge Wasser kommt es zur Bildung öliger Tropfen, die theils an der Oberfläche schwimmen, theils am Boden liegen. Fügt man mehr Wasser hinzu und kocht, so geht das Oel in Lösung. Beim Erkalten trübt sich die Flüssigkeit milchig, wird aber, je nachdem sich Krystalle ausscheiden, wieder klar. Die mehrfach aus Wasser umkrystallisirte Substanz zeigte im Vacuum über Schwefelsäure keinen 
Gewichtsverlust. Die Analyse ${ }^{1}$ ) stimmte gut auf die Formel des Benzoylleucins.

$0,1715 \mathrm{~g}$ Substanz gaben $0,4184 \mathrm{~g} \mathrm{CO}_{2}$ und $0,1145 \mathrm{~g} \mathrm{H}_{2} \mathrm{O}$.

$0,2191 \mathrm{~g}$ Substanz verbrauchten $9,3 \mathrm{ccm}$., ${ }^{1 / 10}$ Normalsäure.

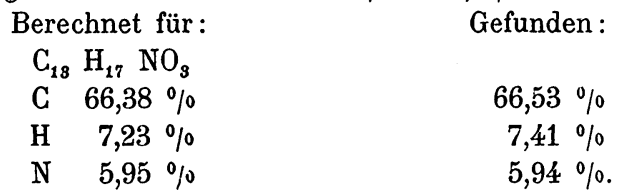

Der Schmelzpunkt liegt bei $135-140^{\circ}$, d. h. die Substanz beginnt bei $135^{\circ} \mathrm{zu}$ schmelzen und ist bei $140^{\circ}$ flüssig. E. Fischer gibt $126-128^{\circ}$ an. Im Reagensrohr vorsichtig erhitzt, schmilzt die Substanz zu einem klaren farblosen Oel, das bei weiterem Erhitzen unzersetzt sublimirt und sich an den kälteren Theilen des Glases als Oel niederschlägt. Das Produkt verlangt zur Lösung 690 Theile Wasser von $19^{\circ}$. Die Lösung wurde durch mehrstündiges Schütteln der in Wasser suspendirten fein zerriebenen Substanz im Schüttelapparat hergestellt. Es ist ferner löslich in Alkohol, Aether, Essigäther, Chloroform, schwer löslich in Benzol, unlöslich in Ligroin und Petroläther. Das Benzoylleucin war in alkalischer Lösung optisch-inactiv. Eine Lösung, welche in $26 \mathrm{ccm} .3,0051 \mathrm{~g}$ Substanz und die für 2 Moleküle berechnete Menge $\mathrm{KOH}$ enthielt, also fast $12 \%$ ig war, zeigte keine Einwirkung auf das polarisirte Licht. Das Benzoylleucin bildet mit Basen eine Reihe theilweise gut krystallisirender Salze.

Das Silbersalz wurde durch Eintragen von frisch gefälltem Silberoxyd in eine kochend heisse Lösung der freien Säure gewonnen. Aus dem Filtrat schieden sich beim Erkalten weisse schön ausgebildete Krystalle ab (sechsseitige längliche Blättchen). Durch Umkrystallisiren aus heissem Wasser, in dem es leicht löslich ist, wurde es gereinigt. Im Trockenschrank bei $100^{\circ}$ erhitzt, erleidet es weder Veränderung noch Wasserverlust.

1) Die Kohlenstoff- und Wasserstoffbestimmungen wurden im offenen Rohr unter Benutzung von Bleichromat, die Stickstoffbestimmungen nach Kjeldahl ausgeführt. 
0,2955 g Substanz gaben 0,0936 g metall. Silber.

Berechnet:

Ag $31,67 \%$
Gefunden: $31,64 \%$.

Bleisalz. Zur Darstellung dieses Salzes wurde Bleicarbonat in eine heisse wässerige Lösung von Benzoylleucin eingetragen und die Mischung einige Zeit im Sieden erhalten. Die filtrirte und etwas eingeengte Lösung scheidet allmählich farblose Krystalle ab. Dieselben sind in einzelnen Drusen angeordnet und bestehen aus radiär gestellten dicht neben einander liegenden Blättchen oder Nadeln. Im Wasser sind sie schwer löslich. Längeres Erhitzen bei $100^{\circ}$ bewirkt keine Gewichtsabnahme. Die Bestimmung des Bleis wurde nach Berzelius ausgeführt.

$$
\begin{array}{cr}
0,204 \mathrm{~g} \text { Substanz gaben } 0,0621 \mathrm{~g} \mathrm{~Pb} . \\
\text { Berechnet: } & \text { Gefunden: } \\
\mathrm{Pb} 30,66 \% & 30,48 \% .
\end{array}
$$

Das Zinksalz liess sich durch Kochen der wässerigen Lösung des Benzoylleucins mit Zinkcarbonat darstellen. Die Krystalle waren in Wasser leicht löslich und enthielten kein Krystallwasser.

$$
\begin{array}{cr}
0,238 \mathrm{~g} \text { Substanz gaben } 0,0370-\mathrm{g} \mathrm{ZnO} . \\
\text { Berechnet: } & \text { Gefunden: } \\
\mathrm{Zn} 12,19 \% & 12,43 \%
\end{array}
$$

Das Baryumsalz, durch Kochen der wässerigen Lösung mit Baryumcarbonat gewonnen, ist in Wasser ausserordentlich leicht löslich und konnte nicht krystallinisch erhalten werden. Beim Verdunsten der wässerigen Lösung hinterblieben harte Krusten, die zerrieben ein weisses Pulver darstellten. Dasselbe benetzte sich nur schwer mit Wasser, ging jedoch beim Umrühren leicht in Lösung.

Auch das Kalisalz, durch Sättigen der Säure mit der berechneten Menge Kalilauge dargestellt, zeichnete sich durch grosse Löslichkeit in Wasser und Alkohol aus und konnte nicht zur Krystallisation gebracht werden. Beim Verdunsten seiner Lösung im Vacuum schieden sich weisse Krusten ab, die an der Luft alsbald zerflossen.

Wässerige Lösungen von Quecksilberchlorid oder Kupfersulfat riefen in der Kalisalzlösung gelblichweisse bezw. grün- 
lichblaue amorphe Niederschläge hervor. Dieselben wurden abfiltrirt, ausgewaschen und mit heissem Wasser behandelt. Dabei gingen sie nicht in Lösung, nahmen aber eine wachsartige Beschaffenheit an. Nach dem Erkalten wurden sie wieder fest, das Kupfersalz unter Veränderung seiner Farbe in Grün. Auch das Kupfersalz, das ich durch Kochen der wässerigen Lösung mit frisch gefälltem Kupferoxydhydrat herstellte, zeigte dasselbe Verhalten.

Schliesslich ist noch zu erwähnen, dass das Benzoylleucin auch auf einem anderen Weg, nämlich durch Erhitzen von Benzoësäure und Leucin im geschlossenen Rohr auf $200^{\circ}$, von Destrem ${ }^{1}$ ) erhalten worden ist. Die Angaben, die Destrem über diese Verbindung macht, sind aber sehr dürftig.

\section{Benzoylasparaginsäure.}

Das Benzoyliren geschah genau in der beim Leucin angegebenen Weise. Zur Isolirung benutzte ich ebenfalls die beiden oben beschriebenen Verfahren. Im ersten betrug die Ausbeute 68\%, im zweiten $74 \%$ der theoretischen Menge. Ein wesentlich besseres Resultat $89 \%$, wurde erhalten, als bei im Uebrigen gleicher Versuchsanordnung statt der Natronlauge Kalilauge zur Anwendung kam. Aus Wasser krystallisirt die Verbindung in schönen grossen Nadeln, die sternförmig zusammengelagert sind. Das Auftreten eines Oels beim Erhitzen mit Wasser wurde nicht beobachtet. Die Krystalle enthalten kein Krystallwasser. Bei der Analyse gaben sie folgende Werthe :

$0,1387 \mathrm{~g}$ Substanz lieferten $0,2835 \mathrm{~g} \mathrm{CO}_{2}$ und $0,0590 \mathrm{~g} \mathrm{H}_{2} \mathrm{O}$.

$0,2040 \mathrm{~g}$ Substanz erforderten $8,2 \mathrm{ccm}$. 1/10 Normalsäure.

Berechnet für $\mathrm{C}_{11} \mathrm{H}_{11} \mathrm{NO}_{5}$ :

Gefunden:

$$
\begin{array}{lr}
\text { C } 55,69 \% & 55,73 \% \\
\text { H } 4 ; 64 \% & 4,72 \% \\
\text { N } 5,90 \% & 5,62 \%
\end{array}
$$

Den Schmelzpunkt fand ich bei $182-183^{\circ}$, E. Fischer giebt $180-181^{\circ}$ an.

Beim Erhitzen im Reagensrohr schmilzt die Verbindung zuerst und färbt sich dann unter Zersetzung und Verkohlung braun, indem sich ein weisses Sublimat von Benzoesäure zeigt

1) Bulletin de la société de Paris, Bd. XXX, 481, Jahrg. 1878. 
und der Bittermandelgeruch des Benzonitrils wahrnehmbar ist. Zur Lösung bedarf die Benzoylasparaginsäure 227 Theile Wasser von $18^{\circ}$, während $\mathrm{E}$. Fischer 261 Theile bei $20^{\circ}$ angiebt. Sie ist in heissem Wasser sehr leicht löslich, leicht löslich ferner in Alkohol, Methylalkohol und Essigäther. Beim Erwärmen löst sie sich in Aceton, Amylalkohol und Eisessig. Unlöslich ist sie in Aether, Chloroform, Petroläther, Ligroin und Benzol.

Zur Bestimmung des Drehungsvermögens benutzte ich eine wässerige Lösung, welche 8,197\% Substanz und die für 4 Moleküle KOH berechnete Menge Kalilauge enthielt. Das specifische Gewicht betrug 1,0881. Die Lösung drehte das polarisirte Licht im $22 \mathrm{dcm}-\mathrm{Rohr}$ bei Natriumlicht um 6,270 nach rechts. Mithin ergibt sich für Benzoylasparaginsäure bei Gegenwart von 4 Molekülen $\mathrm{KOH}(\boldsymbol{\alpha})_{\mathrm{D}}=+34,8$, E. Fischer fand $(\boldsymbol{\alpha})_{\mathrm{D}}=+37,4$. Der von mir gefundene niedrigere Werth erklärt sich vielleicht durch den grösseren Alkaligehalt der von mir benutzten Lösung.

Das Natronsalz, durch Neutralisiren der Süure mit der berechneten Menge Natronlauge hergestellt, scheidet sich nur aus der sehr concentrirten Lösung in Form harter Warzen ab. Es ist in Wasser leicht löslich, ebenso in Alkohol, aus dem es sich beim Verdunsten als krystallinisches Pulver absetzt. Dasselbe besteht aus regellos durcheinander liegenden feinsten Nadeln.
Bei $100^{\circ}$ getrocknet gaben $0,41.00 \mathrm{~g}$ Substanz 0,2091 $\mathrm{g} \mathrm{Na}_{\mathbf{8}} \mathrm{SO}_{4}$. Berechnet : $\mathrm{Na} 16,37 \%$ Gefunden : $16,46 \%$.

Das Kalisalz konnte nur als farbloser Syrup erhalten werden.

Das Silbersalz, durch Kochen mit frisch gefälltem Silberoxyd gewonnen, schied sich aus dem eingeengten Filtrat in Form radiär gestellter, dicht neben einander liegender Nadeln oder Blättchen ab. Es ist in Alkohol unlöslich. Krystallwasser ist nicht vorhanden.

0,1655 g Substanz gaben 0,0791 g metallisches Silber.

Berechnet :

Ag $47,89 \%$

Gefunden :

$47,79 \%$. 
Auch das Kupfersalz krystallisirt gut. Zu seiner Darstellung wurde die wässerige Lösung der freien Säure mit frisch gefälltem Kupferoxyd gekocht. Das blau gefärbte Filtrat lässt nach entsprechender Einengung bis stecknadelkopfgrosse, runde, dunkelgrüne, seidenglänzende Krystallaggregate, welche nicht an der Wandung des Gefässes haften, austreten. Unter dem Mikroskop erkennt man feine Nadeln, welche von einem Mittelpunkt nach allen Seiten ausstrahlen. In Alkohol ist es unlöslich. Beim Erhitzen im Trockenschrank geht die dunkelgrüne Farbe in ein fahles Grün über und gleichzeitig verliert die Substanz zwei Moleküle Wasser.

$0,1695 \mathrm{~g}$ wasserfreies Salz gaben $0,0445 \mathrm{~g} \mathrm{CuO}$.

Berechnet:

Cu $21,14 \%$

\section{Gefunden :}

$20,88 \%$.

Blei-, Baryum- und Zinksalz, durch Kochen mit den Carbonaten hergestellt, konnten nicht zur Krystallisation gebracht werden. Das Bleisalz ist in heissem Wasser sehr schwer, in Alkohol unlöslich. Der aus der wässerigen Lösung abgeschiedene amorphe Niederschlag wurde im Trockenschrank getrocknet und analysirt.

$0,2195 \mathrm{~g}$ Substanz gaben $0,1497 \mathrm{~g} \mathrm{PbSO}_{4}$.

$\begin{array}{rr}\text { Berechnet: } & \text { Gefunden: } \\ \mathrm{Pb} 46,83 \% & 46,56 \% .\end{array}$

Das Baryumsalz ist in Wasser sehr leicht löslich. Die wässerige Lösung wurde durch Alkohol gefällt. Der Niederschlag wurde durch Lösen in Wasser und Fällen durch Alkohol gereinigt und im Trockenschrank getrocknet.

$0,423 \mathrm{~g}$ Substanz ergaben $0,2623 \mathrm{~g} \mathrm{Ba} \mathrm{SO}$.

$$
\begin{array}{lr}
\text { Berechnet: } & \text { Gefunden : } \\
\text { Ba } 36,82 \% & 36,45 \% \text {. }
\end{array}
$$

Das Zinksalz ist in Wasser und Alkohol sehr leicht löslich.

\section{Benzoylglutaminsäure.}

Die ersten Versuche wurden nach dem Verfahren von Baum ausgeführt. Das Produkt, welches nach dem Auskochen mit Petroläther gewonnen wurde, war in Alkohol und Wasser leicht löslich. Beim Verdunsten der alkoholischen Lösung 
hinterblieb ein hellgelbes, zähes Oel, das keine Neigung zum Krystallisiren zeigte. In seiner mit Kalilauge neutralisirten wässerigen Lösung rief salpetersaures Silber eine weisse, krystallinische Fällung hervor. Der Niederschlag wurde abfiltrirt, in Wasser suspendirt und mit Schwefelwasserstoff zerlegt. Beim Einengen des Filtrats blieb ein Syrup zurück, der zuweilen auf Zusatz von Aether zu einer krystallinischen Masse erstarrte, zuweilen aber durchaus nicht krystallisirt erhalten werden konnte. Ein besser krystallisirendes Produkt wurde gewonnen, als ich auf die Glutaminsäure das oben beschriebene Verfahren anwandte. Nachdem aus dem Reactionsgemisch die durch Salzsäure zur Abscheidung gebrachte Benzoesäure abfiltrirt war, schieden sich nach mehrtägigem Stehen prismatische Krystalle der Benzoylglutaminsäure ab. Die Ausbeute betrug $55 \%$ der Theorie.

In einem anderen Versuch wurde nach Beendigung der Benzoylirung und Ansäuern mit Salzsäure mit Aether ausgeschüttelt, der Aether verdunstet, aus dem Rückstand in oben beschriebener Weise die Benzoesäure weggeblasen und die zurückbleibende Masse in warmem Wasser gelöst. Beim Erkalten schied sich zunächst noch etwas Benzoesäure, dann Benzoylglutaminsäure ab. Auch die Mutterlauge lieferte beim Eindampfen noch weitere Krystalle; schliesslich bleibt ein zähes Oel zurück, das nicht mehr krystallisirt. Die Krystalle schmelzen bei $98^{\circ}$. Sie verloren im Vacuum bei einer Temperatur, die dem Schmelzpunkt nahe liegt, ein Molekül Krystallwasser.

$$
\begin{gathered}
0,6305 \mathrm{~g} \text { Substanz verloren } 0,0435 \mathrm{~g} \mathrm{H}_{8} \mathrm{O}, \\
\text { für } \mathrm{C}_{\mathbf{1 8}} \mathrm{H}_{\mathbf{4 9}} \mathrm{NO}_{5}+\mathrm{H}_{8} \mathrm{O}
\end{gathered}
$$

berechnet:

$\mathrm{H}_{2} \mathrm{O} \quad 6,69 \%$

Die krystallwasserfreie Verbindung lieferte folgende Zahlen : 0,1234 g Substanz ergaben $0,2590 \mathrm{~g} \mathrm{CO}_{2}$ und $0,0594 \mathrm{~g} \mathrm{H}_{2} \mathrm{O} .0,189 \mathrm{~g}$ verbrauchten $7,2 \mathrm{ccm} .{ }^{1 / 10}$ Normalsäure.
Berechnet für $\mathrm{C}_{12} \mathrm{H}_{13} \mathrm{NO}_{5}$ :
C. $\quad 57,37 \%$
$\mathrm{H} \quad 5,17 \%$
$\mathrm{N} \quad 5,57 \%$
Gefunden:
$57,21 \%$
$5,34 \%$
$5,33 \%$.

Der Schmelzpunkt der wasserfreien Verbindung liegt bei $152-154^{0}$.

Hoppe-Seyler's Zeitschrift f. physiol. Chemie. XXIX. 
Im Reagensrohr erhitzt verhält sich die Benzoylglutaminsäure ebenso wie Benzoylasparaginsäure. Bei $18^{\circ}$ bedarf sie etwa 95 Theile Wasser zur Lösung. Nach E. Fischer löst sich der Körper in 124 Theilen Wasser von $20^{\circ}$. Er ist leicht löslich in Methyl-, Aethyl- und Amylalkohol, ebenso in Eisessig, Aceton, Essigäther, schwerer löslich in Aether und Chloroform; in Petroläther, Ligroin, Benzol, Benzin unlöslich. Die Benzoylglutaminsäure war in alkalischer Lösung optischinactiv. Eine Lösung, welche in $26 \mathrm{ccm} .2,0922 \mathrm{~g}$ Substanz und die für zwei Moleküle berechnete Menge $\mathrm{KOH}$ enthielt, zeigte keine Einwirkung auf das polarisirte Licht. Die eben beschriebene Säure zeigt ganz dieselben Eigenschaften, Krystallwassergehalt, Schmelzpunkt wie diejenige, welche von E. Fischer bei der Benzoylirung der racemischen Glutaminsäure gewonnen war. Es ist also während des Benzoylirungsprocesses in ausgedehntem Maasse eine Racemisirung eingetreten. Das stimmt genau mit den Angaben von E. Fischer überein, welcher bereits feststellte, dass beim Benzoyliren der natürlichen Glutaminsäure ein Gemenge von optisch-activen und racemischen Benzoylverbindungen entsteht. Vermuthlich lag in dem von mir erhaltenen nicht krystallisirenden Oel die active Form der Benzoylglutaminsäure vor. Vielleicht ist bei der Benzoylirung des Leucins auch eine Racemisirung eingetreten; dafür spricht die optische Inactivität des von mir erhaltenen Benzoylleucins. Eine Entscheidung wird erst getroffen werden können, wenn die Benzoylverbindung des racemischen Leucins vorliegt.

Die Salze der Alkalien und alkalischen Erden sind in Wasser sehr leicht löslich und konnten nicht krystallisirt erhalten werden.

Das Silbersalz fällt als weisser krystallinischer Niederschlag auf Zusatz von Silbernitrat zu der mit Kalilauge neutralisirten Säure. Es wurde aus heissem Wasser umkrystallisirt.

0,2274 g Substanz ergaben 0,105 g met. Silber.

Berechnet:

Gefunden:

Ag $\quad 46,45 \%$

$46,17 \%$. 
Das Zinksalz krystallisirt in feinen farblosen Nadeln. In Wasser ist es schwer löslich, in Alkohol unlöslich.

0,2465 g Substanz ergaben 0,0629 g Zn0. Berechnet:

$\mathrm{Zn} \quad 20,70 \%$

Gefunden:

$20,44 \%$.

Amorph ist das Cadmiumsalz, welches durch Umlegen des Barytsalzes mit Cadmiumsulfat hergestellt wurde.

$0,2375 \mathrm{~g}$ des beim Verdunsten der wässerigen Lösung im Exsiccator zurückbleibenden Salzes gaben 0,0945 g CdS. Berechnet:

Cid $31,02 \%$

Gefunden:

$30,94 \%$.

Ebenfalls amorph ist das in Wasser unlösliche Bleisalz. Durch Auswaschen mit Wasser konnte es gereinigt werden.

Bei $100^{\circ}$ getrocknet gaben 0,3725 g Substanz 0,2463 g $\mathrm{PbSO}_{4}$. Berechnet:

$\mathrm{Pb} \quad 45.39 \%$

Gefunden:

$45,15 \%$.

\section{Dibenzoyltyrosin.}

Bei dem Tyrosin erhielt ich zunächst ein völlig negatives Resultat. Das Verfahren, welches bei den Amidosäuren der Fettreihe zum Ziel geführt hatte, versagte hier, wenigstens gelang es mir nicht, aus dem Reactionsgemisch ein einheitliches Produkt $\mathrm{zu}$ isoliren. Das Ergebniss wurde sofort ein anderes als statt der Natronlauge Kalilauge zur Anwendung kam. Gleich der erste in dieser Weise ausgeführte Versuch ergab eine Ausbeute an Dibenzoyltyrosin von $93 \%$ der Theorie. Die weiteren scheinbar ebenso ausgeführten Benzoylirungen missglückten wieder, bis es mir schliesslich gelang, festzustellen, dass die Bildung nur dann erfolgt, wenn ununterbrochen eine schwach alkalische Reaction ') herrscht. So wie die Reaction auch nur vorübergehend sauer wird, scheidet sich ein reichlicher Niederschlag aus, der auch nach sofortigem Zusatz von Alkali nicht wieder in Lösung geht und dessen Untersuchung noch nicht beendet ist. Nimmt man die Benzoylirung unter den angegebenen Cau-

1) Bei stark alkalischer Reaction scheint ein Benzoesäurerest in die Amidogruppe einzutreten und ein Benzoyltyrosin zu entstehen. Mit der Untersuchung dieses Körpers bin ich noch beschäftigt. 
telen vor, so entsteht regelmässig Dibenzoyltyrosin, allerdings in wechselnden Mengen (35-.93\% der Theorie). Um die Verbindung zu isoliren, wurde die alkalische Flüssigkeit mit Salzsäure versetzt, der entstehende Niederschlag abgesaugt, mit Wasser gewaschen, getrocknet, mit Petroläther ausgekocht, der in Petroläther unlösliche Theil in Alkohol gelöst und die alkoholische Lösung mit Wasser versetzt. Es enstand sofort ein lockerer weisser krystallinischer Niederschlag, der aus verdünntem Alkohol umkrystallisirt wurde. Die Krystalle erscheinen unter dem Mikroskop als feine Nadeln. Krystallwasser ist nicht vorhanden.

$0,1275 \mathrm{~g}$ Substanz gaben $0,3307 \mathrm{~g} \mathrm{CO}_{2}$ und $0,0579 \mathrm{~g} \mathrm{H}_{2} \mathrm{O} .0,205 \mathrm{~g}$ verlangten $5,3 \mathrm{ccm}$. ${ }^{1} / 10$ Normalsäure.

\begin{tabular}{|c|c|c|}
\hline \multicolumn{2}{|c|}{ Berechnet für $\mathrm{C}_{23} \mathrm{H}_{19} \mathrm{O}_{5} \mathrm{~N}$ : } & Gefunden: \\
\hline C & $70,95 \%$ & $70,66 \%$ \\
\hline $\mathrm{H}$ & $4,88 \%$ & $5,04 \%$ \\
\hline $\mathrm{N}$ & $3,59 \%$ & $3,61 \%$. \\
\hline
\end{tabular}

Nach der Analyse liegt ein Dibenzoyltyrosin vor, und zwar ist je ein Benzoesäurerest in die Hydroxyl- und Amidogruppe eingetreten. Dass ersteres der Fall ist, geht daraus hervor, dass die Verbindung beim Kochen mit Millon'schem Reagens sich nicht mehr roth färbt. Der Schmelzpunkt liegt bei $211-212^{\circ}$. Im Reagensrohr vorsichtig erhitzt, schmilzt Dibenzoyltyrosin zuerst und färbt sich dann bräunlich unter Abgabe eines gelblichen Dampfes, der in öligen Tropfen sich wieder an den kälteren Wandungen absetzt. Der Geruch nach bitteren Mandeln ist auch hier deutlich wahrzunehmen.

In kaltem Wasser ist es völlig unlöslich, in heissem lösen sich nur Spuren, leicht löslich in Alkohol, Methylalkohol, Essigäther, Aceton, Eisessig, weniger löslich in Benzol und Aether, unlöslich in Petroläther und Ligroin. Die Substanz dreht rechts, zu einer quantitativen Bestimmung reichte das Material nicht aus.

Das Kalisalz ist in Wasser leicht löslich. Es scheidet sich aus der wässerigen Lösung erst nach starkem Concentriren aus, und zwar als gallertartige Masse. Unter dem Mikroskop erkennt man von einem Centrum aus wellenförmig verlaufende 
lange Fäden. Auch in Alkohol ist es leicht löslich. Beim langsamen Verdunsten des Alkohols krystallisirte es in schönen federförmig angeordneten Nadeln oder Säulen.

Die bei $100^{\circ}$ getrocknete Substanz ergab bei einer Menge von 0,2755 g $0,0548 \mathrm{~g} \mathrm{~K}_{2} \mathrm{SO}_{4}$
Berechnet:
K $9,13 \%$
Gefunden :
$8,89 \%$.

Das Cadmiumsalz fiel auf Zusatz von gelöstem Cadmiumsulfat zur Lösung des Kalisalzes als weisse gallertartige Masse aus. Sie wurde abfiltrirt, gewaschen und in heissem Alkohol gelöst. Es scheiden sich kleine Warzen ab, die einen zusammenhängenden Bodensatz bilden. Mikroskopisch bestehen diese Warzen aus einem Haufwerk langer feinster Nadeln, die eine radiäre Anordnung erkennen lassen, aber auch durcheinander gefilzt sind.

Von dem bei $100^{\circ}$ getrockneten Salz gaben $0,176 \mathrm{~g} \mathrm{0,029} \mathrm{g}$ CdS. Berechnet:

Cd $12,61 \%$

Gefunden:

$12,78 \%$.

Es ist mir eine willkommene Gelegenheit, meinem hochverehrten Lehrer, Herrn Professor Dr. H. Thierfelder, für das bei meiner Arbeit mir bewiesene Wohlwollen und Interesse auch an dieser Stelle meinen herzlichsten Dank auszusprechen. 